doi: 10.2306/scienceasia1513-1874.2013.39S.070

\title{
Confidence intervals for multivariate value at risk
}

\author{
Y.L. Goh ${ }^{\mathrm{a}, *}$, A.H. Pooi ${ }^{\mathrm{b}}$ \\ a Department of Mathematical and Actuarial Sciences, UTAR Complex. Jalan Genting Kelang, \\ 53300 Setapak, Kuala Lumpur, Malaysia \\ b Sunway University Business School. No. 5, Jalan Universiti, Bandar Sunway, 46150 Petaling Jaya, Selangor, \\ Malaysia
}

*Corresponding author, e-mail: gohyl@utar.edu.my, yann.ling@yahoo.com

Received 7 Jan 2013

Accepted 5 Apr 2013

\begin{abstract}
Confidence intervals for the $\gamma$-quantile of a linear combination of $N$ non-normal variates with a linear dependence structure would be useful to the financial institutions as the intervals enable the accuracy of the value at risk (VaR) of a portfolio of investments to be quantified. Here we construct $100(1-\alpha) \%$ confidence intervals for the $\gamma$-quantile using procedures based on bootstrap, normal approximation and hypothesis testing. We show that the method based on hypothesis testing produces a confidence interval which is more satisfactory than those found by using bootstrap or normal approximation.
\end{abstract}

KEYWORDS: non-normal variates, $\gamma$-quantile, bootstrap, hypothesis testing

\section{INTRODUCTION}

Consider a portfolio consisting of $N$ stocks. The absolute value of the $\gamma$-quantile of the return of the portfolio is called the value at risk $(\mathrm{VaR})$ of the portfolio.

VaR has been frequently used by commercial and investment banks to capture the potential loss in value of their traded portfolios from adverse market movements over a specified period.

To evaluate VaR in the multivariate situation where $N$ stocks are involved, we usually begin with the evaluation of a multivariate distribution for the $N$ stocks. A common approach is to fit the data on returns by the multivariate version of the normal, Student t or skewed Student $\mathrm{t}$ distribution. Other approaches may take into account the tail-dependence ${ }^{1}$, and asymmetry ${ }^{2-5}$. A more sophisticated approach is one which is based on copulas ${ }^{2,6-13}$.

Presently we use an approach based on a type of non-normal distribution called the quadratic-normal distribution $^{14,15}$. To describe the approach, we first let $\mathbf{S}=\left(S_{1}, S_{2}, \ldots, S_{N}\right)^{\mathrm{T}}$ be a vector of uncorrelated variates of which $S_{i}$ can be expressed as

$$
S_{i}= \begin{cases}\lambda_{i 1} e_{i}+\lambda_{i 2}\left(e_{i}^{2}-\frac{1}{2}\left[1+\lambda_{i 3}\right]\right), & e_{i} \geqslant 0 \\ \lambda_{i 1} e_{i}+\lambda_{i 2}\left(\lambda_{i 3} e_{i}^{2}-\frac{1}{2}\left[1+\lambda_{i 3}\right]\right), & e_{i}<0\end{cases}
$$

where $e_{i} \sim N(0,1)$. The variate $S_{i}$ is said to have a quadratic-normal distribution with parameters 0 and $\boldsymbol{\lambda}_{i}$, and we may write $S_{i} \sim Q N\left(0, \boldsymbol{\lambda}_{i}\right)$. The mean of
$S_{i}$ is 0 while the $k$ th moment of $S_{i}$ is given by $m_{k}=$ $E\left(S_{i}^{k}\right), k=2,3,4$. The standardized moments

$$
\bar{m}_{3}=m_{3} / m_{2}^{3 / 2}, \quad \bar{m}_{4}=m_{4} / m_{2}^{2}
$$

will then be, respectively, the measures of skewness and kurtosis of $S_{i}$. Next, let $\mathbf{A}$ be an $N \times N$ orthogonal matrix, $\boldsymbol{\mu}$ an $N \times 1$ vector of constants and $\mathbf{R}=\left(R_{1}, R_{2}, \ldots, R_{N}\right)^{\mathrm{T}}$ an $N \times 1$ vector given by

$$
\mathbf{R}=\boldsymbol{\mu}+\mathbf{A S},
$$

$\mathbf{w}=\left(w_{1}, w_{2}, \ldots, w_{N}\right)^{\mathrm{T}}$ a vector of constants with $\sum_{i=1}^{N} w_{i}=1$ and

$$
R=\sum_{i=1}^{N} w_{i} R_{i}
$$

When $\lambda_{i 3}=-1$ and $\lambda_{i 2}$ is large, the distribution of the random variable $S_{i}$ will have fat tails and narrow waist. As the matrix A represents an orthogonal transformation, and the vector $\boldsymbol{\mu}$, on the other hand represents a translation, the distribution of $R_{i}$ will also have fat tails and narrow waist. As the distribution of stock return often also has fat tails and narrow waist, and the returns of different stocks are usually correlated, the distribution of $\mathbf{R}$ given by (1) can be used to model the joint distribution of the returns of $N$ stocks. For a portfolio of $N$ stocks, the portfolio return can be represented by $R$ given by (2).

Let $F_{R}$ be the cumulative distribution function of $R$ and assume that the $\gamma$-quantile, $Q_{\gamma}=F_{R}^{-1}(\gamma)$, is 
uniquely defined. When $\gamma$ is small, the absolute value of $Q_{\gamma}$ will represent the VaR which has a confidence level of $100(1-\gamma) \%$.

After finding an estimate for the VaR, it is usually desirable to access the accuracy of the VaR estimate by constructing a confidence interval for the VaR.

The layout of the paper is as follows. In the next three sections, we describe, respectively, the procedures based on bootstrap, normal approximation and hypothesis testing for finding a confidence interval for the VaR. We then compare the performance of the three methods for constructing confidence intervals for the VaR. In the last section, we give an example which shows that multivariate quadratic-normal distribution is able to fit a real data set obtained from the Kuala Lumpur stock exchange.

\section{BOOTSTRAP CONFIDENCE INTERVAL FOR $\gamma$-QUANTILE}

First, let $\left(r_{1 j}, r_{2 j}, \ldots, r_{N j}\right)$ be the $j$ th observed value of $\mathbf{R}, j=1,2, \ldots, n$. From the $n$ observed values $\left(r_{1 j}, r_{2 j}, \ldots, r_{N j}\right), j=1,2, \ldots, n$, we first compute the $(k, l)$ entry of the matrix $\hat{\mathbf{V}}$ of the estimated variance-covariance of $\mathbf{R}$ as shown below: $\hat{v}_{k l}=(1 / n) \sum_{j=1}^{n} r_{k j} r_{l j}-\hat{\mu}_{k} \hat{\mu}_{l}$ where $\hat{\mu}_{k}=$ $(1 / n) \sum_{j=1}^{n} r_{k j}$.

We next compute $\hat{\mathbf{A}}=\left[\hat{\mathbf{a}}_{1} \hat{\mathbf{a}}_{2} \cdots \hat{\mathbf{a}}_{N}\right]$ where $\hat{\mathbf{a}}_{i}$ is the $i$ th eigenvector of $\hat{\mathbf{V}}$, and $\left\|\hat{\mathbf{a}}_{i}\right\|=1$. By using $\hat{\mathbf{A}}$, we compute

$$
\left(\begin{array}{c}
s_{1 j} \\
s_{2 j} \\
\vdots \\
s_{N j}
\end{array}\right)=\hat{\mathbf{A}}^{\mathrm{T}}\left(\begin{array}{c}
r_{1 j}-\hat{\mu}_{1} \\
r_{2 j}-\hat{\mu}_{2} \\
\vdots \\
r_{N j}-\hat{\mu}_{N}
\end{array}\right), j=1,2, \ldots, n .
$$

By using the constrained maximum likelihood procedure ${ }^{16}$, we find the quadratic-normal distributions $Q N\left(0, \hat{\boldsymbol{\lambda}}_{i}\right)$ and $Q N(\hat{\mu}, \hat{\boldsymbol{\lambda}})$ which fit $s_{i 1}, s_{i 2}, \ldots, s_{i n}$ and the $n$ observed values of $R$. Let $z_{\gamma}$ be the $(1-\gamma)$-quantile of the standard normal distribution. An estimate of the $\gamma$-quantile of $R$ is then given by

$$
\hat{Q}=\hat{\mu}+\hat{\lambda}_{1}\left(-z_{\gamma}\right)+\hat{\lambda}_{2}\left[\hat{\lambda}_{3}\left(-z_{\gamma}\right)^{2}-\frac{1+\hat{\lambda}_{3}}{2}\right] .
$$

Next we generate $B$ values of $\left(\tilde{r}_{1 j}, \tilde{r}_{2 j}, \ldots, \tilde{r}_{N j}\right)$, $(j=1,2, \ldots, n)$, using

$$
\left(\begin{array}{c}
\tilde{r}_{1 j} \\
\tilde{r}_{2 j} \\
\vdots \\
\tilde{r}_{N j}
\end{array}\right)=\left(\begin{array}{c}
\hat{\mu}_{1} \\
\hat{\mu}_{2} \\
\vdots \\
\hat{\mu}_{N}
\end{array}\right)+\hat{\mathbf{A}}\left(\begin{array}{c}
\tilde{s}_{1 j} \\
\tilde{s}_{2 j} \\
\vdots \\
\tilde{s}_{N j}
\end{array}\right)
$$

where $\tilde{s}_{i j} \sim Q N\left(0, \hat{\boldsymbol{\lambda}}_{i}\right), j=1,2, \ldots, n ; i=$ $1,2, \ldots, N$

By using the constrained maximum likelihood procedure, we find the quadratic-normal distribution $Q N(\tilde{\mu}, \tilde{\boldsymbol{\lambda}})$ which fits the values $\tilde{r}_{j}=\sum_{i=1}^{N} w_{i} \tilde{r}_{i j}, j=$ $1,2, \ldots, n$. Next let

$$
\tilde{Q}=\tilde{\mu}+\tilde{\lambda}_{1}\left(-z_{\gamma}\right)+\tilde{\lambda}_{2}\left[\tilde{\lambda}_{3}\left(-z_{\gamma}\right)^{2}-\frac{1+\tilde{\lambda}_{3}}{2}\right]
$$

be the estimated quantile, and $Q N\left(\tilde{\mu}^{*}, \tilde{\lambda}^{*}\right)$ the quadratic-normal distribution which fits the $B$ values of $\tilde{Q}$.

The approximately-100(1- $\alpha) \%$ bootstrap confidence interval for the $\gamma$-quantile is then given by $\left[Q_{\mathrm{L}}, Q_{\mathrm{U}}\right]$ where

$Q_{\mathrm{L}}=\tilde{\mu}^{*}+\tilde{\lambda}_{1}^{*}\left(-z_{\alpha / 2}\right)+\tilde{\lambda}_{2}^{*}\left[\tilde{\lambda}_{3}^{*}\left(-z_{\alpha / 2}\right)^{2}-\frac{1+\tilde{\lambda}_{3}^{*}}{2}\right]$

and

$$
Q_{\mathrm{U}}=\tilde{\mu}^{*}+\tilde{\lambda}_{1}^{*}\left(z_{\alpha / 2}\right)+\tilde{\lambda}_{2}^{*}\left[\left(z_{\alpha / 2}\right)^{2}-\frac{1+\tilde{\lambda}_{3}^{*}}{2}\right] .
$$

\section{CONFIDENCE INTERVALS BASED ON NORMAL APPROXIMATION}

From the $B$ values $\tilde{Q}^{(1)}, \tilde{Q}^{(2)}, \ldots, \tilde{Q}^{(B)}$ of $\tilde{Q}$ we can find the estimated variance

$$
\tilde{\sigma}^{2}=\frac{1}{B-1} \sum_{b=1}^{B}\left(\tilde{Q}^{(b)}-\overline{\tilde{Q}}\right)^{2}
$$

where $\overline{\tilde{Q}}=(1 / B) \sum_{b=1}^{B} \tilde{Q}^{(b)}$. Then the approximately-100 $(1-\alpha) \%$ confidence interval based on normal approximation for the $\gamma$-quantile is

$$
\left[\hat{Q}-z_{\alpha / 2} \tilde{\sigma}, \hat{Q}+z_{\alpha / 2} \tilde{\sigma}\right] .
$$

\section{PROCEDURE BASED ON HYPOTHESIS} TESTING

Consider the problem of testing $H_{0}: Q_{\gamma}=Q_{\gamma}^{0}$ against $H_{1}: Q_{\gamma} \neq Q_{\gamma}^{0}$. Suppose we test the above $H_{0}$ by using the decision rule of accepting $H_{0}$ at the $\alpha$ level if $Q_{\mathrm{L}}^{(0)} \leqslant \hat{Q} \leqslant Q_{\mathrm{U}}^{(0)}$ where $Q_{\mathrm{L}}^{(0)}$ and $Q_{\mathrm{U}}^{(0)}$ 
are, respectively, the $100(\alpha / 2) \%$ and $100(1-\alpha / 2) \%$ points of the quadratic-normal distribution which is used to fit the $B$ values of $\tilde{Q}$ obtained when the $B$ values of $\left(\left(\tilde{r}_{1 j}, \tilde{r}_{2 j}, \ldots, \tilde{r}_{N j}\right), j=1,2, \ldots, n\right)$ are generated using

$$
\left(\begin{array}{c}
\tilde{r}_{1 j} \\
\tilde{r}_{2 j} \\
\vdots \\
\tilde{r}_{N j}
\end{array}\right)=\left(\begin{array}{c}
\mu_{1}^{(m)} \\
\mu_{2}^{(m)} \\
\vdots \\
\mu_{N}^{(m)}
\end{array}\right)+\mathbf{A}^{(m)}\left(\begin{array}{c}
\tilde{s}_{1 j} \\
\tilde{s}_{2 j} \\
\vdots \\
\tilde{s}_{N j}
\end{array}\right)
$$

where $\tilde{s}_{i j} \sim Q N\left(0, \boldsymbol{\lambda}_{i}^{(m)}\right), j=1,2, \ldots, n$ and $\left(\left(\left(\mu_{i}^{(m)}, \boldsymbol{\lambda}_{i}^{(m)}\right), i=1,2, \ldots, N\right), \mathbf{A}^{(m)}\right)$ is found as follows.

Firstly, for a given value of $\left(\left(\mu_{i}, \boldsymbol{\lambda}_{i}\right), i=\right.$ $1,2, \ldots, N), \mathbf{A})$, we find the moment $m_{k}=$ $E(R-E(R))^{k}, \quad k=2,3,4$. Let $(\mu, \boldsymbol{\lambda})$ be such that $\mu=E(R)$ and the $k$ th central moment of the quadratic-normal distribution $Q N(\mu, \boldsymbol{\lambda})$ is equal to $m_{k}, k=2,3,4$. Then $R$ is approximately distributed as $Q N(\mu, \boldsymbol{\lambda})$. Finally, $\left(\left(\left(\mu_{i}^{(m)}, \boldsymbol{\lambda}_{i}^{(m)}\right), i=1,2, \ldots, N\right), \mathbf{A}^{(m)}\right)$ is $\left(\left(\left(\mu_{i}, \boldsymbol{\lambda}_{i}\right), i=1,2, \ldots, N\right), \mathbf{A}\right)$ which minimizes

$D^{2}=(\mu-\hat{\mu})^{2}+\left(\lambda_{1}-\hat{\lambda}_{1}\right)^{2}+\left(\lambda_{2}-\hat{\lambda}_{2}\right)^{2}+\left(\lambda_{2} \lambda_{3}-\hat{\lambda}_{2} \hat{\lambda}_{3}\right)^{2}$

subject to

$$
\mu+\lambda_{1}\left(-z_{\gamma}\right)+\lambda_{2}\left[\lambda_{3}\left(-z_{\gamma}\right)^{2}-\frac{1+\lambda_{3}}{2}\right]=Q_{\gamma}^{0} .
$$

An approximately-100 $(1-\alpha) \%$ confidence interval for the $\gamma$-quantile of $R$ is now given by $\left\{Q_{\gamma}^{0}:\right.$ The null hypothesis that $Q_{\gamma}=Q_{\gamma}^{0}$ is accepted at the $\alpha$ level\}.

\section{NUMERICAL EXAMPLES}

Fig. 1 shows 100 simulated bootstrap confidence intervals for the $\gamma$-quantile of $R$ when $n=50, \mu_{1}=$ $0, \boldsymbol{\lambda}_{1}^{\mathrm{T}}=(0.32,0.68,0.065), \mu_{2}=0, \boldsymbol{\lambda}_{2}^{\mathrm{T}}=$ $(0.378,0.639,0.073)$,

$$
\mathbf{A}=\left(\begin{array}{cc}
0.3090 & 0.9511 \\
-0.9511 & 0.3090
\end{array}\right)
$$

The upper limits of the 100 confidence intervals have been arranged in ascending order.

Figs. 2 and 3 show 100 possible confidence intervals based on normal approximation and hypothesis testing. As in Fig. 1, the upper limits of the 100 confidence intervals have been arranged in ascending order.

Figs. 1-3 show that the estimated coverage probability of the confidence interval based on hypothesis

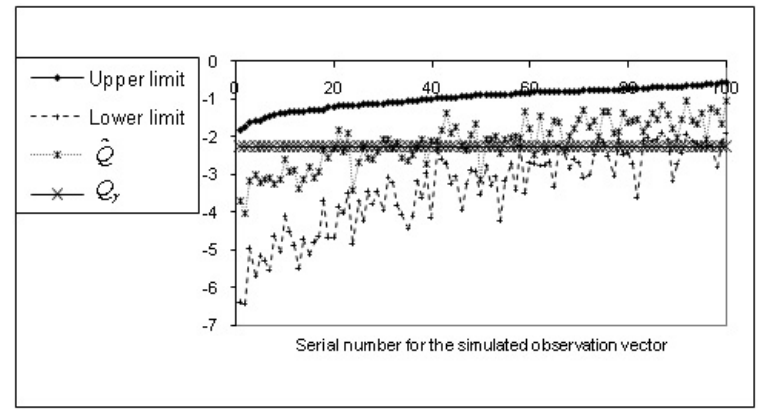

Fig. 1100 simulated bootstrap confidence intervals for $\gamma$ quantile when $\gamma=0.01, \alpha=0.05, n=50, B=$ 100. Estimated coverage probability: 0.82 ; average length: 2.395, $\hat{Q}$ : estimate of $\gamma$-quantile; $Q_{\gamma}$ : true value of $\gamma$ quantile.

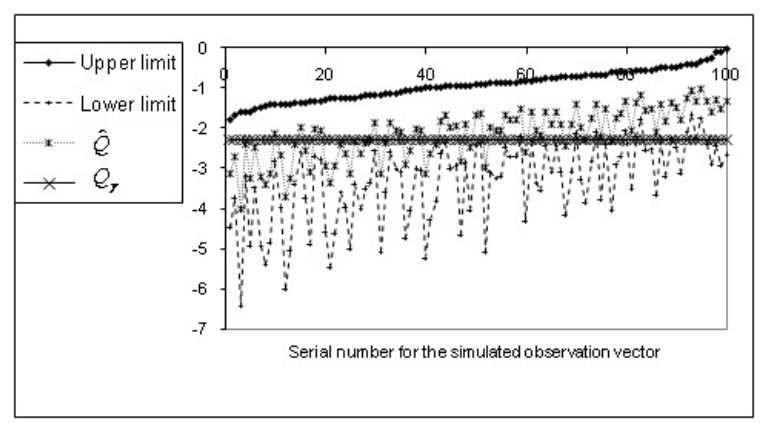

Fig. 2100 simulated confidence intervals based on normal approximation for $\gamma$-quantile. Estimated coverage probability: 0.89 , average length: 2.4296 .

testing is closer to the target value 0.95 than those of the bootstrap confidence interval and the confidence interval based on normal approximation.

Further comparison of the 3 types of confidence

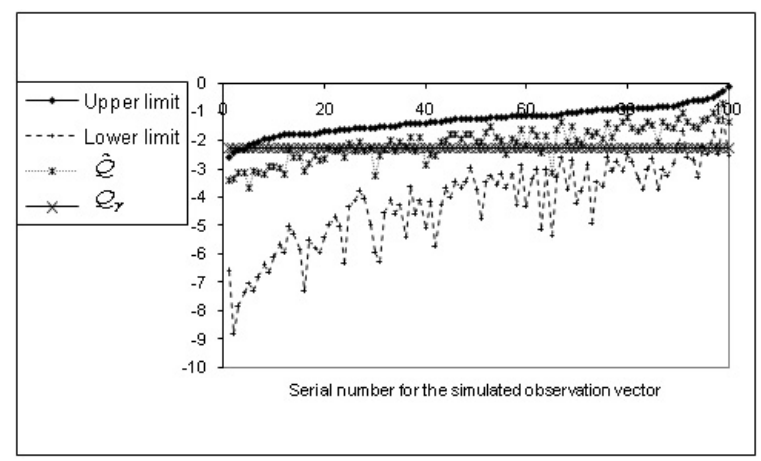

Fig. 3100 simulated confidence intervals based on hypothesis testing procedure for $\gamma$-quantile. Estimated coverage probability: 0.91 , average length: 2.9261 . 
Table 1 Estimated coverage probabilities and average lengths of confidence intervals for $\gamma$-quantile $(\gamma=0.01$, $\left.\alpha=0.05, \mu_{1}=\mu_{2}=0, n=50\right) . \quad N=B=100$, standard error of the estimated coverage probability $\approx$ 0.0218 .

\begin{tabular}{ccccccc}
\hline No & BTP & NAP & HTP & BTL & NAL & HTL \\
\hline 1 & 0.82 & 0.89 & 0.91 & 2.39207 & 2.42961 & 2.918 \\
2 & 0.8 & 0.8 & 0.88 & 2.45177 & 2.49157 & 3.019 \\
3 & 0.79 & 0.82 & 0.93 & 2.31436 & 2.35217 & 2.9195 \\
4 & 0.72 & 0.72 & 0.83 & 2.17881 & 2.21492 & 2.634 \\
5 & 0.91 & 0.89 & 0.94 & 0.76893 & 0.76353 & 1.214 \\
6 & 0.85 & 0.86 & 0.91 & 1.26915 & 1.29416 & 1.8135 \\
7 & 0.79 & 0.85 & 0.87 & 1.81021 & 1.83762 & 2.248 \\
8 & 0.9 & 0.9 & 0.93 & 0.81293 & 0.8142 & 1.3455 \\
9 & 0.71 & 0.69 & 0.82 & 2.16665 & 2.20639 & 2.5805 \\
10 & 0.77 & 0.75 & 0.85 & 1.22067 & 1.24205 & 1.7185 \\
\hline
\end{tabular}

$\mathrm{BTP}=$ Estimated coverage probability of confidence interval based on bootstrap.

$\mathrm{NAP}=$ Estimated coverage probability of confidence interval based on normal approximation.

HTP=Estimated coverage probability of confidence interval based on hypothesis testing.

$\mathrm{BTL}=$ Average length of confidence interval based on bootstrap.

$\mathrm{NAL}=$ Average length of confidence interval based on normal approximation.

HTL=Average length of confidence interval based on hypothesis testing.

intervals can be found in Table 1 which displays the estimated coverage probabilities and average lengths for 10 values of $\left(\mu_{1}, \boldsymbol{\lambda}_{1}, \mu_{2}, \boldsymbol{\lambda}_{2}, \mathbf{A}\right)$. The 10 values of $\boldsymbol{\lambda}_{1}$ and $\boldsymbol{\lambda}_{2}$ are displayed in Table 2. The measures of skewness and kurtosis $\left(\bar{m}_{3}\right.$ and $\left.\bar{m}_{4}\right)$ of the quadraticnormal distribution with the given $\boldsymbol{\lambda}_{i}$ are also included in Table 2. Table 1 shows that the coverage probability of the confidence interval based on hypothesis testing is closer to the target value 0.95 than those of the bootstrap confidence interval and confidence interval based on normal approximation.

Table 1 also shows that the average length of the confidence interval based on the hypothesis testing is longer than those of the bootstrap confidence interval and confidence interval based on normal approximation. This is not surprising because in order to have a larger coverage probability, the length of the confidence interval should be made longer.

\section{APPLICATIONS IN FINANCE}

The random variables $R_{1}, R_{2}, \ldots, R_{N}$ in the first section may be considered to be the returns of $N$ stocks, and the $\gamma$-quantile $Q_{\gamma}$ of $R$ becomes the value
Table 2 The parameters and measures of skewness and kurtosis of the quadratic-normal distribution.

\begin{tabular}{cccccc}
\hline No & $\bar{m}_{3}$ & $\bar{m}_{4}$ & & $\lambda_{1}^{\mathrm{T}}$ & \\
& & & & $\lambda_{2}^{\mathrm{T}}$ & \\
\hline 1 & 3 & 16.6 & 0.322184 & 0.680924 & 0.065316 \\
& 2.8 & 15 & 0.377794 & 0.638861 & 0.072964 \\
2 & 3.4 & 20.2 & 0.190061 & 0.770776 & 0.02769 \\
& 2.6 & 13.4 & 0.450006 & 0.589852 & 0.115554 \\
3 & 3 & 16.7 & 0.300017 & 0.688155 & 0.020679 \\
& 2.4 & 12 & 0.502252 & 0.547528 & 0.125795 \\
4 & 3.2 & 18.4 & 0.247697 & 0.72906 & 0.029551 \\
& 1 & 4.4 & 0.955589 & 0.177217 & 0.88736 \\
5 & -0.2 & 2.4 & 1.20867 & -0.17517 & -0.52415 \\
& 2 & 9.4 & 0.62038 & 0.454663 & 0.189261 \\
6 & 0.6 & 3.1913 & 1.0745 & 0.055572 & 2.958 \\
& 1.6 & 7.2 & 0.732179 & 0.359491 & 0.270896 \\
7 & 2 & 9.3 & 0.603592 & 0.45809 & 0.151303 \\
& 0.4 & 2.8 & 1.11827 & -0.00217 & -71.0956 \\
8 & -0.4 & 2.8 & 1.11826 & -0.15408 & -0.01403 \\
& 0.8 & 3.8 & 0.99794 & 0.126695 & 1.16716 \\
9 & 3.6 & 22.6 & 0.054687 & 0.838405 & -0.08815 \\
& 0.4 & 2.7 & 1.15398 & -0.02222 & -8.11265 \\
10 & -1 & 10.6 & 0.171503 & 0.387604 & -1.47341 \\
& 1.4 & 6.2 & 0.797129 & 0.305546 & 0.361719 \\
\hline
\end{tabular}

at risk (VaR) of the portfolio consisting of these $N$ stocks. Thus if we can show that $\mathbf{R}$ can be written as $\mathbf{R}=\boldsymbol{\mu}+\mathbf{A S}$ of which $S_{1}, S_{2}, \ldots, S_{N}$ are uncorrelated and $S_{i} \sim Q N\left(0, \boldsymbol{\lambda}_{i}\right)$, then the methods in the second and fourth sections can be applied to find confidence intervals for the VaR of the portfolio.

In the following analysis, the data obtained from the Kuala Lumpur Stock Exchange (KLSE) are used. The data are the daily stock prices of three companies, namely Genting Bhd., Gamuda Bhd. and Tanjong PLC Bhd. in the KLSE from Thomson Financial Datastream $(01 / 01 / 1993$ to $31 / 8 / 2002)$. The data for the period from 01/07/1997 to 30/06/1999 are excluded in the present investigation because these data were collected during the financial crisis in SE Asia. The following results in the forms of table and figure are extracted from Ref. 15.

The variance-covariance matrix associated with the portfolio is

$$
\left(\begin{array}{lll}
4.6316 & 0.7453 & 1.2520 \\
0.7453 & 4.0142 & 1.2299 \\
1.2520 & 1.2299 & 5.7027
\end{array}\right) .
$$

Fig. 4 shows that the distribution of the portfolio returns $R^{\mathrm{P}}$ can be approximated well using the quadratic-normal distribution. Thus the methods in the second and fourth sections may be used to find confidence intervals for the VaR of the portfolio. 


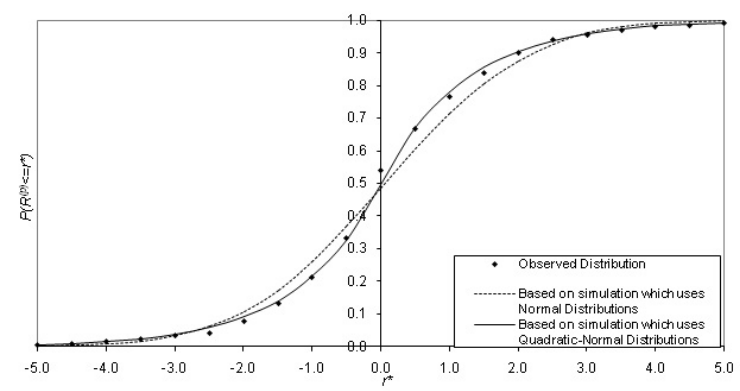

Fig. 4 Cumulative distribution of return for the portfolio.

\section{REFERENCES}

1. Sibuya M (1959) Bivariate extreme statistics, I. Ann Inst Stat Math 11, 195-210.

2. Patton AJ (2006) Modelling asymmetric exchange rate dependence. Int Econ Rev 47, 527-56.

3. Erb CB, Harvey CR, Viskanta TE (1994) Forecasting international equity correlations. Financial Analysts Journal, 32-45.

4. Longin F, Solnik B (2001) Extreme correlation of international equity markets. J Finance 56, 649-76.

5. Ang A, Bekaert G (2002) International asset allocation with regime shifts. Rev Financ Stud 15, 1137-87.

6. Sklar M (1959) Fonctions de répartition à $n$ dimensions et leurs marges. Publ Inst Statist Univ Paris 8, 11.

7. Patton AJ (2006) Estimation of multivariate models for time series of possibly different lengths. J Appl Econometrics 21, 147-73.

8. Ausin MC, Lopes HF (2010) Time-varying joint distribution through copulas. Comput Stat Data Anal 54, 2383-99.

9. Jondeau E, Rockinger M (2006) The copula-garch model of conditional dependencies: An international stock market application. J Int Money Finance 25, 827-53.

10. Fischer M, Köck C, Schlüter S, Weigert F (2009) An empirical analysis of multivariate copula models. Quant Finance 9, 839-54.

11. Genest C, Ghoudi K, Rivest LP (1995) A semiparametric estimation procedure of dependence parameters in multivariate families of distributions. Biometrika $\mathbf{8 2}$, 543-52.

12. Chen X, Fan Y (2006) Estimation and model selection of semiparametric copula-based multivariate dynamic models under copula misspecification. $J$ Econometrics 135, 125-54.

13. Min A, Czado C (2010) Bayesian inference for multivariate copulas using pair-copula constructions. $J \mathrm{Fi}$ nanc Econometrics 8, 511-46.

14. Pooi AH (2003) Effects of non-normality on confidence intervals in linear models. Tech rep, Univ of Malaya.

15. Yap VC (2004) Development of value at risk (VaR) models with applications to Malaysian capital market. $\mathrm{PhD}$ thesis, Multimedia Univ.

16. Goh YL, Pooi AH (2005) Estimation of parameters of the quadratic-normal distribution. Tech rep, Univ of Malaya. 